\title{
EVALUATION OF PANCREATICO-BILIARY DISEASE BY MRCP
}

\author{
Shivanand S. Melkundi ${ }^{1}$, Shrishail Patil2 ${ }^{2}$ Krishna Chaitanya Nunna ${ }^{3}$
}

${ }^{1}$ Professor, Department of Radiodiagnosis, MR Medical College, Gulbarga.

${ }^{2}$ Professor, Department of Radiodiagnosis, MR Medical College, Gulbarga.

${ }^{3}$ Post Graduate, Department of Radiodiagnosis, MR Medical College, Gulbarga.

\section{ABSTRACT}

MRCP means magnetic resonance cholangio-pancreatography. First described in clinical practice in 1991. MRCP technique is based on heavily T2 weighted pulse sequences, which result in dramatic increase in contrast between stationary fluid (bile) and background tissue.

\section{OBJECTIVES OF STUDY}

- $\quad$ To describe features of pancreaticobiliary diseases on MRCP.

- Outlining the extent in terms of involvement of adjacent structures, vessels and soft tissues.

- To help in deciding further course of management.

- To identify the anatomical variants.

- $\quad$ Comparing MRCP to ERCP whenever necessary.

- To prove the Magnetic Resonance Cholangio-Pancreatography (MRCP) is one of the best imaging modality for evaluation of pancreatico-biliary disease.

\section{MATERIAL AND METHODS}

The present study was undertaken to evaluate the role of MRCP in evaluation of pancreaticobiliary diseases. The study will be done on patients presenting with features suggestive of pancreatico-biliary diseases attending the OPD or admitted in various wards of Basaveshwar Teaching and General Hospital and Sangameshwar Teaching Hospital, Kalaburagi, attached to M.R. Medical College, Kalaburagi. A total of 50 patients were included in our study.

\section{RESULT}

Majority of patients in study population were males (58\%), while $42 \%$ were females. The mean age of the study sample was 46.6 years and maximum numbers of cases were observed in 30-70 years of age group. Benign causes were seen in 11-50 years of age group, while malignant causes were more common between 41-75 years of age group. Majority of pathologies observed were benign $38(76 \%)$. Most common benign disorder observed was cholelithiasis with choledocholithiasis (20\%) followed by acute pancreatitis (12\%). Majority of CBD strictures were benign and commonly seen in females (8\%). CBD strictures were more commonly observed in the distal common bile duct. Malignant pathologies were observed in 24\% patients. Most common malignant pathology seen was cholangiocarcinoma (12\%) followed by periampullary carcinoma (6\%) and gallbladder carcinoma (6\%).

\section{CONCLUSION}

MRCP is non-invasive, non-ionizing imaging method for evaluation of pancreatico-biliary anatomy and pathology. It is superior diagnostic modality in detection and characterization of pancreatico-biliary pathologies.

\section{KEYWORDS}

MRCP; CBD Strictures; Cholangiocarcinoma; Cholelithiasis.

HOW TO CITE THIS ARTICLE: Melkundi SS, Patil S, Nunna KC. "Evaluation of pancreatico-biliary disease by MRCP.” Journal of Evolution of Medical and Dental Sciences 2015; Vol. 4, Issue 104, December 28; Page: 16929-16934,

DOI: $10.14260 /$ jemds/2015/2551

\section{INTRODUCTION}

MRCP means magnetic resonance cholangio-pancreatography. First described in clinical practice in 1991 (Wallner et al. 1991). ${ }^{1}$ MRCP technique is based on heavily T2 weighted pulse sequences, which result in dramatic increase in contrast

Financial or Other, Competing Interest: None.

Submission 14-12-2015, Peer Review 15-12-2015,

Acceptance 24-12-2015, Published 26-12-2015.

Corresponding Author:

Dr. Shivanand S. Melkundi,

Department of Radiology,

Mahadevappa Rampure Medical College,

Gulbarga.

E-mail: shivanandmelkundi@gmail.com

DOI:10.14260/jemds/2015/2551 between stationary fluid (Bile) and background tissue (Hepatic, pancreatic parenchyma and peritoneal fat). ${ }^{2}$

Background is sufficiently suppressed to result in a cholangiographic or pancreaticographic effect without need for contrast medium administration as in other invasive cholangiographies, which results in a very high signal intensity of bile and background at low. ${ }^{3}$

In initial attempts to visualize biliary tree, steady-state free precession imaging was used with high contrast over several breath holds. ${ }^{4,5}$ However, steady-state free-precession sequences proved too sensitive to signal loss from magnetic susceptibility, fluid motion and pulsation. So now-a-days preferred imaging is Rapid Acquisition with Relaxation Enhancement (RARE). ${ }^{6}$ and Fast Spin-Echo (FSE). ${ }^{2,7,8}$ 
sequences. With long effective echo times (TE), only fluid-filled compartments are observed with nearly complete background suppression and minimal sensitivity to motion and signal loss due to magnetic susceptibility. This is the advantage of fast spin-echo sequence over steady-state free precession. ${ }^{3}$

\section{AIMS AND OBJECTIVES}

- To describe features of pancreaticobiliary diseases on MRCP.

- Outlining the extent in terms of involvement of adjacent structures, vessels and soft tissues.

- To help in deciding further course of management.

- To identify the anatomical variants.

- Comparing MRCP to ERCP whenever necessary.

- To prove the Magnetic Resonance CholangioPancreatography (MRCP) is one of the best imaging modality for evaluation of pancreaticobiliary disease.

\section{MATERIALS AND METHODS}

The present study was undertaken to evaluate the role of MRCP in evaluation of pancreaticobiliary diseases. The study will be done on patients presenting with features suggestive of pancreaticobiliary diseases attending the OPD or admitted in various wards of Basaveshwar Teaching and General Hospital and Sangameshwar Teaching Hospital, Kalaburagi, attached to M.R. Medical College, Kalaburagi. A total of 50 patients were included in our study.

\section{Study Design}

A prospective study.

\section{Source of Data}

Patients referred to the Department of Radiodiagnosis, Basaveswar Teaching and General Hospital Kalaburagi for MRCP.

\section{Sample Size}

The study comprised a total of fifty patients of pancreaticobiliary disease.

\section{Inclusion Criteria}

All patients who are detected to have any pancreaticobiliary diseases on MRCP.

\section{RESULTS}

- A total of 50 patients, suspected clinically for pancreaticobiliary diseases underwent MRCP examination were chosen for the purpose of the study. Majority of patients in study population were males (58\%), while $42 \%$ were females.

- Pain in abdomen, jaundice, nausea and vomiting were the most frequent presenting complaints while fever, loss of appetite, steatorrhoea, constipation and distension of abdomen were less common. Most of patients presented with combination of symptoms (76\%).

- The mean age of the study sample was 46.6 years with a range of 0-100 years. Maximum numbers of cases were observed in 30-70 years of age group. Majority of benign causes were seen in 11-50 years of age group, while malignant causes were more common between 41-75 years of age group.
- Majority of pathologies observed were benign 38 (76\%). Most common benign disorder observed was cholelithiasis with choledocholithiasis (20\%) followed by acute pancreatitis $(12 \%)$. Cholelithiasis with choledocholithiasis was seen predominantly in males; acute pancreatitis was seen predominantly in males. Least commonly observed benign pathologies were one case of postoperative stricture and one case of calcified of hydatid cyst.

- Majority of CBD strictures were benign and commonly seen in females (8\%). CBD strictures were more commonly observed in the distal common bile duct.

- Choledochal cyst was commonly detected in 1st and 2nd decade of life. Four cases of choledochal cyst was observed. All the cases were of type I choledochal cyst.

- Choledocholithiasis, cholelithiasis and cholecystitis were observed in both male and female patients.

- Malignant pathologies were observed in $24 \%$ patients. Most common malignant pathology seen was Cholangiocarcinoma (12\%) followed by periampullary carcinoma $(6 \%)$ and gallbladder carcinoma (6\%). Gallbladder carcinoma and periampullary carcinoma were more predominant in males. Cholangiocarcinoma shows equal distribution among males and females in this study. Most commonly observed cholangiocarcinoma was hilar cholangiocarcinoma (6\%). Least commonly observed malignant pathology was one $(2 \%)$ case of distal CBD cholangiocarcinoma, which was found in female patient.

\section{DISCUSSION}

Evaluation of suspected pancreaticobiliary pathologies has traditionally involved varieties of modalities including Ultrasonography (USG), Computed Tomography (CT) and Invasive Cholangiography (ERCP and PTC). These techniques have limitations because of poor visualization of ductal stones and strictures on USG and CT and need for invasive procedures like ERCP and PTC. MRCP is non-invasive modality that provide good visualization of hepatobiliary and pancreatic ductal system.

Out of 50 patients evaluated, 29 (58\%) patients were male and 21 (42\%) patients were female. The mean age of study population was 46.6 (Range 0-100 yrs). Majority of benign causes were seen in 11-50 yrs of age, while malignant causes were more common between 41-75 yrs of age.

Upadhaya et al. ${ }^{9}$ studied 100 patients, out of which $46 \%$ were male and $54 \%$ were female. Ferrari et al. ${ }^{10}$ studied 131 patients; distribution of male patients in Ferrari et al. 10 was $47 \%$ while that of female was $53 \%$. Soto et al. ${ }^{11}$ studied 43 patients, out of which $47 \%$ were male and $53 \%$ were female.

Miyazaki et al. ${ }^{12}$ studied 56 patients, out of which $66 \%$ were male patients and $34 \%$ were female patients. In our study of 50 patients, $58 \%$ were male and $42 \%$ were female patients. Percentage distribution of male-to-female is more in our study. From above table sex distribution in our study closely matches with Miyazaki et al.

Regarding clinical symptoms, most common clinical presentation in our study was pain in abdomen seen in 44 (88\%) patients followed by jaundice seen in 24 (48\%) patients, while least common presentation was constipation seen in $7(14 \%)$ patients. Almost all patients presented with combination of symptoms. Schwartz et al. ${ }^{13}$ in his study reported that most common presentation was jaundice seen in $68 \%$ patients followed by pain in abdomen seen in $25 \%$ 
patients. This may be because of dedicated study of malignancy as well as less sample size as compared to our study. In our study percentage of pain in abdomen (88\%) was more, this may be because of inclusion of almost all benign and malignant pathologies including pancreatitis.

In our study isolated choledocholithiasis was seen in 5 $(10 \%)$ cases, cholelithiasis in 4 ( $8 \%$ cases) and combination of cholelithiasis with choledocholithiasis was seen in 10 (20\%) patients, out of which majority were male. MRCP clearly demonstrated IHBR dilatation, caliber of CBD, exact location and size of calculus which is difficult to visualize on ultrasound.

Ability to detect bile duct stones at CT depends on a number of factors related to the stone (Size, shape, position, density), bile duct (Dilated vs non-dilated), technology used (Conventional vs helical CT), technique used (Slice thickness, reconstruction interval, pitch, $\mathrm{kVp}$, administration of contrast material), pure cholesterol stones are iso- or slightly hypoattenuating relative to bile, making them difficult, if not impossible, to detect.

This imposes a theoretic upper limit for the CT detectability of choledocholithiasis of approximately $80 \%$. Heavily calcified stones are relatively easily identified, whereas soft-tissue density stones can be iso-attenuating to surrounding tissue, making them difficult to identify. The attenuation of biliary stones varies with their composition. ${ }^{14}$

In our study percentage of cholelithiasis with choledocholithiasis was $20 \%$ while isolated cholelithiasis was seen in $8 \%$ and isolated choledocholithiasis was seen in $10 \%$ cases. Shadan et al.15 reported choledocholithiasis with cholelithiasis in $20 \%$ cases. Macaulay et al. ${ }^{16}$ reported choledocholithiasis in $14.2 \%$ cases. Upadhaya et al. ${ }^{9}$ reported choledocholithiasis in $32 \%$ cases. Reinhold et al. reported choledocholithiasis in $25 \%$ cases. Total no. of choledocholithiasis in our study was 15 (30\%), which matches with Upadhaya et al. Difference in study may be due to difference in sample size.

In 15 cases of CBD calculi seen in our study, the most common location of CBD calculus was distal CBD seen in 11 $(22 \%)$ cases, which is followed by mid-CBD calculi seen in 2 (4\%) cases, which is followed by proximal CBD calculi seen in $2(4 \%)$ cases. On MRCP, CBD stones are seen as hypointense filling defects within lumen of CBD on T2W SE images. Advantage of MRCP is that stones as small as $3 \mathrm{~mm}$ can be visualized. ${ }^{17}$

In our study, CBD strictures were detected in $8(16 \%)$ cases, out of which $5(10 \%)$ were benign, $3(6 \%)$ were malignant. Shadan et al. ${ }^{15}$ reported benign strictures in $4 \%$ cases and postoperative anastomotic stricture in $2 \%$ cases. Bhatt et al. ${ }^{18}$ reported benign strictures in $4 \%$ cases, malignant in $8 \%$ and postoperative anastomotic stricture in $4 \%$ cases. Upadhaya et al. ${ }^{9}$ reported benign CBD stricture in 6\%, malignant in $9 \%$ and postoperative stricture in $6 \%$ cases. Hurter et al. ${ }^{19}$ reported benign stricture in $9.6 \%$ cases and malignant stricture in $5.7 \%$ cases. Percentage of CBD strictures in our study matches with Hurter, et al.

On Ultrasound it is difficult to visualize distal CBD, this problem get solved on MRCP. MRCP demonstrates exact location, length as well as type of stricture.

In $4(8 \%)$ cases of choledochal cyst seen in our study, MRCP yielded diagnostic information by providing exact anatomical map for pre-surgical evaluation. Kim et al. ${ }^{20}$ in his study of 20 patients concluded the same. Upadhaya et al. ${ }^{9}$ reported choledochal cyst in 3\% of cases in his study. All the cases were of type I choledochal cyst.

In our study, pancreatitis was seen in $9(18 \%)$ patients. Out of 9 cases, 7 (14\%) were male suggesting male predilection. This may be because of alcoholism, which is one of causative factor for pancreatitis.

Pattern of MPD varied in all these patients of pancreatitis. Dilated smooth MPD was seen in $4(8 \%)$ cases, dilated tortuous MPD was seen in $2(4 \%)$ cases, ruptured in $1(2 \%)$ cases and was normal in $2(4 \%)$ cases. MPD can remain non-dilated in cases of acute pancreatitis, while in all 3 cases of chronic pancreatitis it is dilated.

Shadan et al. ${ }^{15}$ reported chronic pancreatitis in $10 \%$ cases. Tamura et al. ${ }^{21}$ reported overall sensitivity and specificity values of MRCP for delineating pathologic pancreatic changes were $88 \%$ and $98 \%$, respectively.

We had $1(2 \%)$ case of pancreatitis in male child, USG was normal; however, MRCP showed congenital abnormality of Pancreas Divisum. This patient presented with pain in abdomen. Manfredi et al. ${ }^{22}$ reported Pancreas Divisum in 5\% cases of 107 patients suspected of pancreatic diseases at MRCP 108. This may be due to more sample size and only inclusion criteria of pancreatitis cases in Manfredi et al.

Cholelithiasis was detected in $4(8 \%)$ cases, out of which $2(4 \%)$ were female and $2(4 \%)$ were male. Out of $6(12 \%)$ patients of cholangiocarcinoma evaluated, $3(6 \%)$ were male, while $3(6 \%)$ were female suggesting equal preponderance. Most common type of cholangiocarcinoma seen in our study was Hilar (Klatskin's) tumor seen in $3(6 \%)$ cases. Other types were distal CBD (Extrahepatic) cholangiocarcinoma seen in 1 (2\%) cases and intrahepatic (Peripheral) cholangiocarcinoma seen in $2(4 \%)$ cases. Shadan et al. ${ }^{15}$ reported cholangiocarcinoma in $4 \%$ cases, Bhatt et al. ${ }^{18}$ reported Klatskin's tumor in $12 \%$ cases.

Reinhold. ${ }^{23}$ et al. reported cholangiocarcinoma in $2.3 \%$ cases. Cholangiocarcinoma is primarily tumor of elderly with peak prevalence in 7 th decade and slight male predilection. In our study, majority of cases of cholangiocarcinoma were seen in 6-7th decade.

MRI helps in defining level of obstruction, extent of tumor and staging for pre-surgical evaluation. In some case there is involvement of GB fossa as well as hilar region by mass lesion, in such cases it becomes difficult to define whether CA GB extending to hilar region or it is Primary Hilar Cholangiocarcinoma, so this becomes limiting factor for MRCP.

Out of total $3(6 \%)$ cases of periampullary carcinoma diagnosed on MRCP, 2 (4\%) cases were male and $1(2 \%)$ case was female suggesting male predilection. In our study, majority of cases were between $3 \mathrm{rd}$ and 4 th decade. Shadan et al. reported periampullary CA in $2 \%$ cases. Bhatt et al. reported Periampullary CA in $4 \%$ cases. This may be possible due to less sample size (50 patients) in both above mentioned authors.

Periampullary carcinomas arise within $2 \mathrm{~cm}$ of the major papilla in the duodenum and include four different types of malignancies, namely those originating from (a) the ampulla of Vater itself, (b) the intrapancreatic distal bile duct, (c) the head and uncinate process of the pancreas, and (d) the duodenum. CA head of pancreas is associated with dilatation of both CBD and PD called as "Double duct" sign. Overall, survival is highest for patients with ampullary and duodenal cancers, intermediate for patients with bile duct cancers and lowest for those with pancreatic cancers. ${ }^{24-26}$ 
CA GB was seen in $3(6 \%)$ cases in our study. Shadan et al. ${ }^{15}$ reported CA GB in $4 \%$ cases, which are closely consistent with our findings, while Bhatt et al. reported it in $2 \%$ cases. MRI helps in defining extent, local spread for pre-surgical evaluation.

Bhatt et al.18 in his study of 50 cases reported cholangiocarcinoma in $12 \%$ cases, CA GB in $2 \%$ cases and periampullary CA in $4 \%$ cases. Shadan et al. ${ }^{15}$ in his study of 50 cases reported cholangiocarcinoma in $4 \%$ cases, CA pancreas in $8 \%$ cases, CA GB in $4 \%$ cases and periampullary CA in $4 \%$ cases.

Schwartz et al.13 in his study of 32 cases reported cholangiocarcinoma in $21.8 \%$ cases, CA pancreas in $37.5 \%$ cases, CA GB in $28.1 \%$ cases and periampullary CA in $6.2 \%$ cases. Soto et al. in his study of 43 cases reported cholangiocarcinoma in $13.9 \%$ cases, CA pancreas in $18.6 \%$ cases, CA GB in $4.6 \%$ cases and periampullary CA in $9.3 \%$ cases.

Upadhaya et al. in his study of 100 cases reported cholangiocarcinoma in $9 \%$ cases, CA pancreas in $9 \%$ cases, CA GB in $19 \%$ cases and periampullary CA in $10 \%$ cases. In our study of 50 cases, cholangiocarcinoma was seen in $6(12 \%)$ cases, periampullary CA in $3(6 \%)$ cases and CA GB in $3(6 \%)$ cases.

Overall, percentage distribution of malignant pathologies in our study closely matches with Bhatt et al. and Shadan et al. Larger percentage in Upadhaya et al. may be due to slightly larger sample size (100 patients).

In our study, final diagnostic criteria is histopathology and postoperative findings. In our study of 50 cases surgery/histopathological correlation was done in 50 cases. In 9 cases of pancreatitis, histopathological correlation was not possible as histopath/surgery is not advisable in these patients. Out of 50 cases, 2 cases were non-operable tumors. In these cases diagnosis was confirmed by FNAC.

Depending upon these 56 cases in which surgery/histopathological/FNAC correlation was done, following statistical values are derived.

In our study sensitivity, specificity and likelihood ratio for choledocholithiasis was $45 \%, 97 \%$ and 4 respectively on USG, sensitivity, specificity and likelihood ratio for choledocholithiasis was $80 \%, 92 \%$ and 8 respectively on CT while that on MRCP was $92 \%, 97 \%$ and 9 respectively. Sensitivity, specificity and likelihood ratio was more on MRCP; hence, it is clear that MRCP is more likely to detect choledocholithiasis than USG and CT.

Sensitivity and specificity for cholecystitis in our study was $45.5 \%$ and $100 \%$ on USG, 60 and $100 \%$ on CT, while that on MRCP was $63 \%, 100 \%$ respectively. Likelihood ratio cannot be calculated as specificity is $100 \%$. From this it is clear that MRCP is more sensitive in detection of cholecystitis than USG and CT, while specificity of both USG, CT and MRCP remains same.

Sensitivity, specificity and likelihood ratio for CBD strictures in our study was $40 \%, 55 \%$ and 7 respectively on USG, $65 \%, 80 \%$ and 8 respectively on CT, while that on MRCP was $90 \%, 97 \%$ and 9 respectively. Hence, we can say that MRCP is more likely to detect CBD strictures than USG and CT.

In our study, sensitivity and specificity for choledochal cyst was $100 \%$ and $100 \%$ on USG, CT as well as MRCP. Hence, it is clear that USG, CT and MRCP was similar in detection of choledochal cyst, but MRCP gives better anatomical details.
Sensitivity, specificity and likelihood ratio for malignant pathologies in our study was $73 \%, 84 \%$ and 8 respectively on USG sensitivity, specificity and likelihood ratio was $80 \%, 88 \%$ and 9 respectively on CT while that on MRCP was $96 \%, 94 \%$ and 10 respectively. Hence, we can say that MRCP is more likely to detect pancreaticobiliary malignancies than USG and CT.

\section{CONCLUSION}

MRCP is non-invasive, non-ionizing imaging method for evaluation of pancreaticobiliary anatomy and pathology. It is superior diagnostic modality in detection and characterization of pancreaticobiliary pathologies.

MRCP with its high resolution, multiplanar imaging and 3D reconstruction capability is effective investigation for detection of pancreaticobiliary pathologies.

MRCP with additional MRI sequences (Pre- and postcontrast T1) due to its multiplanar capability, superior soft tissue contrast, better definition of internal architecture allowed better characterization of pancreaticobiliary pathologies.

MRCP is able to detect exact location of biliary obstruction and cause of biliary obstruction.

Use of contrast enhanced MRI (Pre- and post-contrast T1W) with MRCP improves the diagnosis of malignant pathologies and allows safe surgical management decisions by defining extent of malignant lesions.

Potentially useful in patients undergoing biliary enteric anastomosis for knowing the level and extent of strictures.

Pure cholesterol stones are iso- or slightly hypoattenuating relative to bile, making them difficult to detect on CT. Stones as small as $3 \mathrm{~mm}$ are visualized on MRCP.

The addition of T1-weighted gradient echo in phase images to standard MRCP sequences improves the detection and differentiation of hepatolithiasis and intrahepatic pneumobilia.

Malignant strictures are differentiated from benign strictures by specific MR imaging findings. For evaluation of pancreas, 2D and 3D MRCP images provide complementary data. So it is recommended using a combination of both these techniques.

MRCP with additional MRI sequences (Pre- and postcontrast $\mathrm{T} 1 \mathrm{~W}$ ) proved to be a sensitive, non-invasive imaging modality that helps in detection, characterization, evaluation of the pancreaticobiliary diseases. It provides valuable information of diagnostic, therapeutic, prognostic significance and safe surgical management decisions.

\section{REFERENCES}

1. Wallner BK, Schumacher KA, Weidenmaier $\mathrm{W}$, et al. Dilated biliary tract: evaluation with MR cholangiography with a T2-weighted contrast-enhanced fast sequence. Radiology 1991;181:805-808.

2. Ahmet Mesrur Halefoglu, et al. Magnetic resonance cholangiopancreatography: A useful tool in the evaluation of pancreatic and biliary disorders. World J Gastroenterol 2007;13(18):2529-2534.

3. Caroline Reinhold, Patrice M Bret, Laurent Guibaud, et al. MR cholangiopancreatography: Potential and clinical applications. Radiographics 1996;16:309-320.

4. Morimoto K, Shimoi M, Shirakawa T, et al. Biliary obstruction: evaluation with three dimensional MR cholangiography. Radiology 1992;183:578-580. 
5. Ishizaki $\mathrm{Y}$, Wakayama $\mathrm{T}$, Okada $\mathrm{Y}$, et al. Magnetic resonance cholangiography for evaluation of obstructive jaundice. Am J Gastroenterol 1993;88:2072-2077.

6. Hennig J, Nauerth A, Friedburg H, et al. RARE imaging: a fast imaging method for clinical MR. Magn Reson Med 1986;3:823-833.

7. Takehara Y, Ichijo K, Tooyama N, et al. Breath-hold MR cholangiopancreatography with a long-echo-train, fast spin-echo sequence and a surface coil in chronic pancreatitis. Radiology 1994;192:73-78.

8. Lefèvre F, Crouzet P, Gaucher H, et al. Single shot fast spin echo sequence MRI cholangiopancreatography. J Radiol 1998;79(5):415-25.

9. Upadhaya V, Upadhaya DN, Ansari MA, et al. Comparative assessment of imaging modalities in Biliary Obstruction. Ind J Radiol Imag 2006;16:4:577-582.

10. Francesco Saverio Ferrari, Federica Fantozzi, Laura Tasciotti, et al. US, MRCP, CCT and ERCP: A comparative study in 131 patients with suspected biliary obstruction. Med Sci Monit 2005;11(3):MT8-18.

11. Soto JA, Alvarez O, Lopera JE, et al. Biliary obstruction: findings at MR cholangiography and cross-sectional MR imaging. Radiographics 2000;20:353-366.

12. Toshiyuki Miyakazi, Yasuyuki Yamashita, Tadatoshigame. MR cholangiopancreatography using HASTE (HalfFourier Acquisition Single-Shot Turbo Spin-Echo) Sequences. AJR 1996;166:1297-1303.

13. Lawrence H Schwartz, Fergus V Coakley, Yi Sun, et al. Neoplastic pancreaticobiliary duct obstruction: Evaluation with breath-hold MR cholangiopancreatography. AJR 1998;170:1491-1495.

14. Syochum and Bary. Essentials of skeletal radiology 2nd edition. 1996;2:212-235.

15. Andleeb Shadan, Malik GM, Kamili MMA, et al. Role of MRCP in evaluation of suspected biliary and pancreatic diseases. JK-Practitioner 2011;16(1-2):20-25.

16. Shane E Macaulay, Scott J Schulte, John H Sekijima, et al. Evaluation of a non-breath-hold MR cholangiography technique. Radiology 1995;196:227-232.

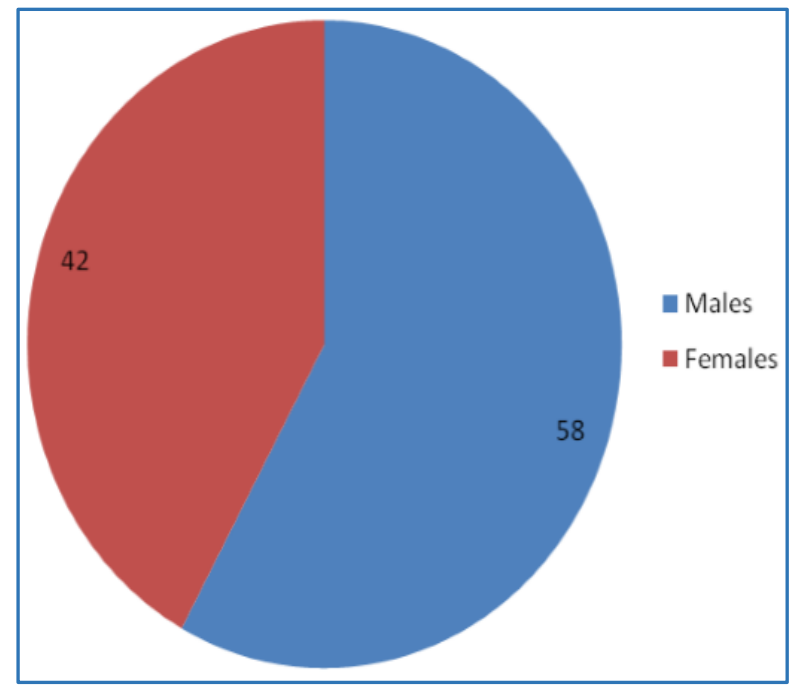

Fig.1: Sex-wise Distribution in the Pancreatico-Biliary Diseases
17. Tublin M, Tessler F, Cheng SL. Effect of injection rate of contrast medium on pancreatic and hepatic helical CT. Radiology 1999;210:97-101.

18. Bhatt C, Shah PS, Prajapati HJ, et al. Comparison of diagnostic accuracy between USG and MRCP in biliary and pancreatic pathology. Indian J Radiol Imaging 2005;15:2:177-181.

19. Hurter D, De Vries C, Potgieter PH, et al. Accuracy of MRCP compared with ERCP in diagnosis of bile duct disorders. SA Journal of Radiology April 2008;14-22.

20. Kim, Donald G, Mitchell Katsuyoshi Ito, et al. Biliary Dilatation: Differentiation of benign and malignant causes- value of adding conventional MR imaging to MR cholangio-pancreaticography. Radiology 2000;214:173181.

21. Ryo Tamura, Tadashi Ishibashi, Shoki Takahashi. Chronic pancreatitis: MRCP versus ERCP for Quantitative Caliber Measurement and Qualitative Evaluation. Radiology 2006;238:3:920-928.

22. Riccardo Manfredi, Guido Costamagna, Maria Gabriella, et al. Pancreas divisum and "Santorinicele": Diagnosis with dynamic MR cholangio-pancreatography with secretin stimulation. Radiology 2000;217:403-408.

23. Bloom CM, Langer B, Wilson SR. Role of US in detection, characterization and staging of cholangiocarcinoma. Radiographics 1999;19:1999-1218.

24. Freeman JS Jr. Cancer: principles and practice of oncology. 6th ed. Philadelphia, PA: Lippincott Williams \& Wilkins, 2001;1126-1217.

25. Chan C, Herrera MF, De La Garza L, et al. Clinical behaviour and prognostic factors of periampullary adenocarcinoma. Ann Surg 1995;5:632-637.

26. Yeo CJ, Sohn TA, Cameron JL, et al. Periampullary adenocarcinoma: analysis of 5-year survivors. Ann Surg 1998;227:821-83.

27. Hee Sun Park, et al. Preoperative evaluation of bile duct cancer: MRI combined with MR cholangiopancreatography versus MDCT with direct cholangiography 2007. DOI:10.2214/AJR.07.2310.

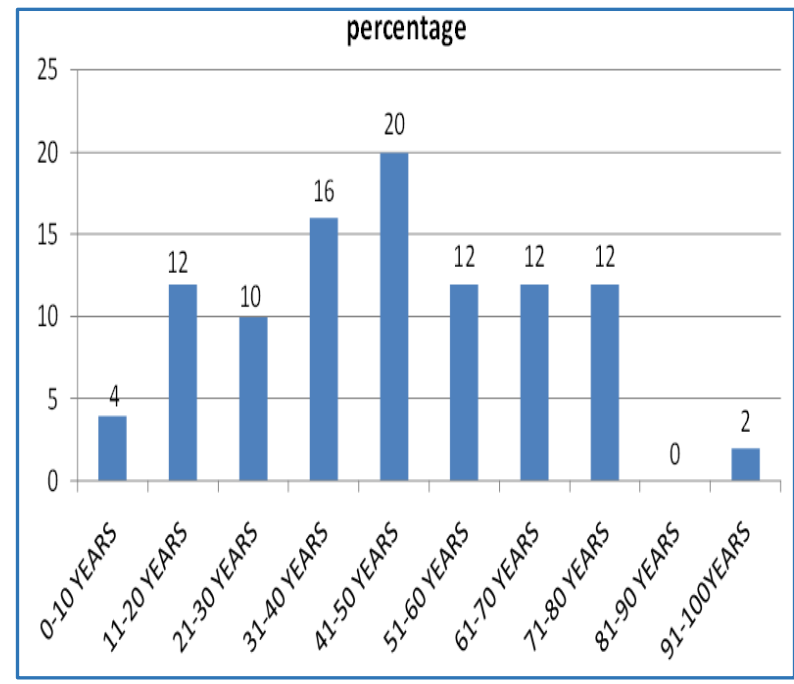

Fig. 2: Age-wise distribution in Pancreatico-Biliary Diseases 


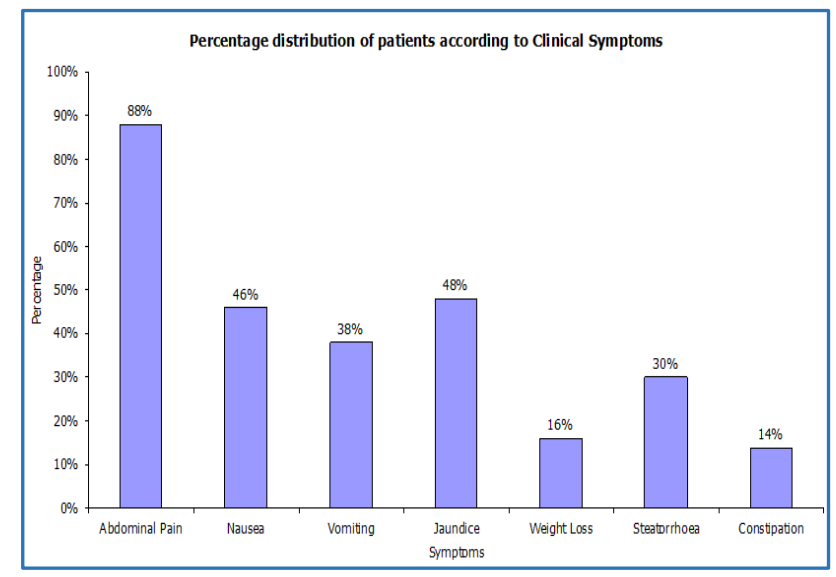

Fig. 3: Clinical Symptoms presented by a patient with various Pancreatico-Biliary Diseases

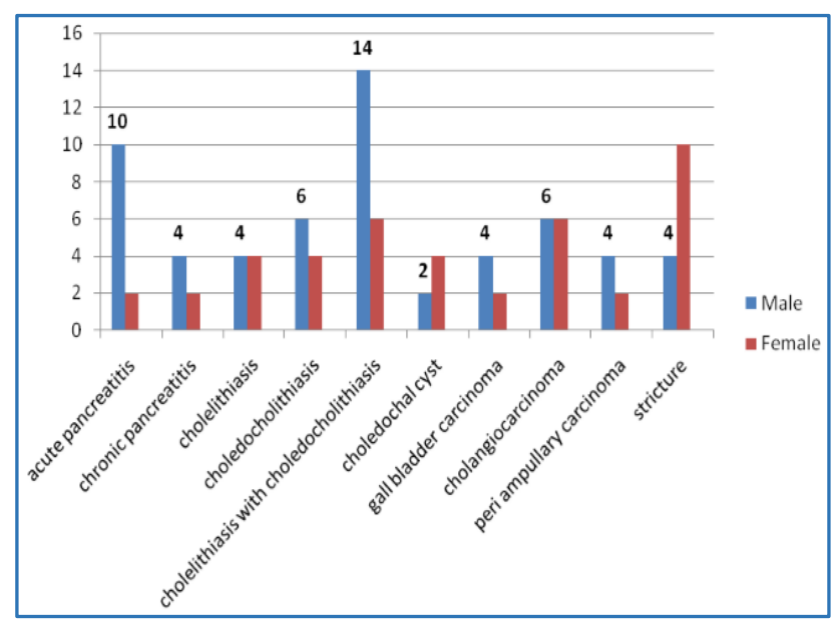

Fig. 4: Sex-wise distribution of various Diseases as Observed on MRCP

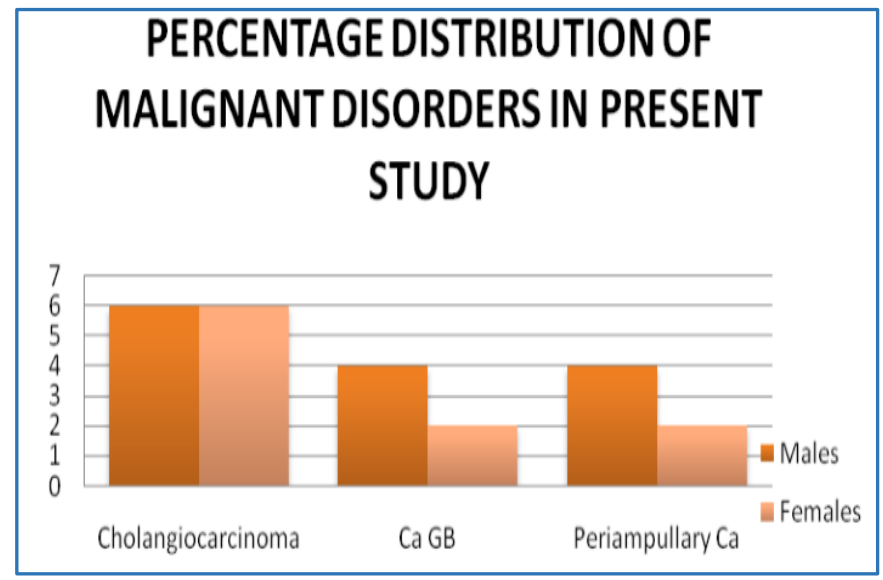

Fig. 5: Distribution of various Malignant Disorders

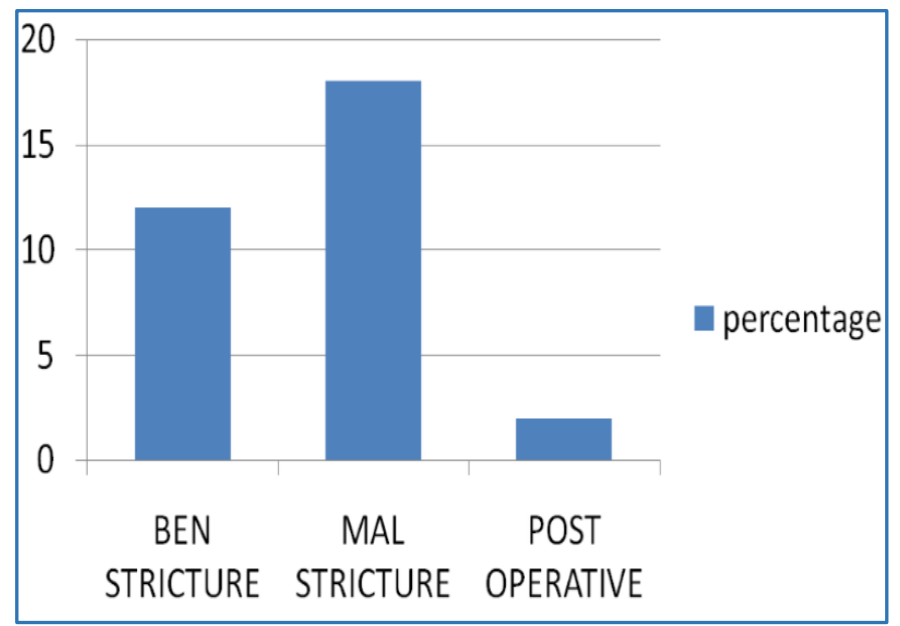

Fig. 6: Showing distribution of Strictures 